\title{
Improvement of ventricular function in systolic heart failure patients with oral L-citrulline supplementation
}

\author{
Karla Balderas-Muñoz ${ }^{1}$, Lilia Castillo-Martínez ${ }^{1}$, Arturo Orea-Tejeda ${ }^{1}$, \\ Oscar Infante-Vázquez ${ }^{2}$, Marcelo Utrera-Lagunas ${ }^{1}$, Raúl Martínez-Memije ${ }^{2}$, \\ Candace Keirns-Davis ${ }^{3}$, Bryan Becerra-Luna ${ }^{2}$, Gabriela Sánchez-Vidal ${ }^{1}$ \\ ${ }^{1}$ Heart Failure Clinic at Instituto Nacional de Ciencias Médicas y Nutrición "Salvador Zubirán”, Mexico, Mexico \\ ${ }^{2}$ Instrumentation Department, Instituto Nacional de Cardiología "ICh”, Mexico, Mexico \\ ${ }^{3}$ Massachusetts General Hospital Interpreter Services, Boston, MA, USA
}

\begin{abstract}
Background: The utility of L-arginine and L-citrulline in treatment of arterial hypertension by increasing vascular oxide nitric availability has been demonstrated. Photoplethysmography, a simple and low-cost optical technique, makes it possible to assess vascular function and to detect changes in blood flow, pulse and swelling of the microvascular tissular space. The aim of the study was to evaluate the effect of L-citrulline supplementation on functional class, ejection fraction and peripheral blood flow in patients with systolic heart failure.

Methods: Thirty-five stable outpatients attending the Heart Failure Clinic at the INCMNSZ underwent clinical evaluation, radioisotopic ventriculography and photoplethysmography before and at the end of 4 months. They were randomized into two groups: experimental group, with oral $L$-citrulline supplementation ( $3 \mathrm{~g} /$ day, $n=20)$ and control group, without supplementation $(n=15)$.

Results: In the experimental group the left ventricular ejection fraction (LVEF) increased $20.3 \%$ at rest and $12.7 \%$ with stress, as well as the right ventricular ejection fraction at rest of $15.10 \%$ and $14.88 \%$ with stress. In addition, functional class improved in 35\%, and the maximum amplitude time/total time (MAT/TT) index decreased 23.1\%. These changes were statistically significant compared with the control group.

Conclusions: Citrulline supplementation significantly improved the LVEF, the endothelial function (MAT/TT index) and functional class. Citrulline can be an important co-adjuvant in the treatment of stable and stable systolic heart failure patients. (Cardiol J 2012; 19, 6: 612-617)

Key words: citrulline, systolic heart failure, endothelial function
\end{abstract}

\section{Introduction}

Heart failure (HF) is a clinical syndrome resulting from damage to myocardial fibers. The main causes are acute or chronic ischemia, hypertension and arrhythmias [1]. There are several molecular mechanisms involved, including apoptosis, oxidative stress, inflammation, endothelial dysfunction and cardiac remodeling [2, 3]. HF is associated with increased activity of angiotensin-converting en-

Address for correspondence: Dr. Arturo Orea-Tejeda, Heart Failure Clinic Director INCMNSZ, Providencia 1218-A Int. 402, Colonia del Valle Delegación Benito Juárez 03100, México, D.F. México, tel/fax: (5255) 55-13-93-84,

e-mail: oreatart@gmail.com

Received: 03.07.2012 Accepted: 06.09.2012 
zyme (ACE) and diminished peripheral blood flow, both of which diminish synthesis of nitric oxide (NO) and increase endothelin-1 [2, 3].

$\mathrm{NO}$ is responsible for maintaining regulated vasodilatation [4]. NO originates with amino acids from the diet (glutamine and glutamate) that, through several reactions in the intestine, are transformed into citrulline. After its absorption, it arrives at the kidney, where the enzymes arginossuccinate synthase and lyase transform it into arginine, which is converted into NO plus citrulline. The citrulline can be transformed in arginine again, providing a recycling axis [4-9] it has been hypothesized that because of the efficiency of the recycling systems the amount of NO produced exceeds the quantity of citrulline formed at a rate of 8:1 [8].

Arginine is the supplement more frequently used, but the drawback is that its hepatic metabolism consumes a great part of it [4]. Glutamine supplements have been studied, but only $27 \%$ is converted into citrulline [10]. Van de Poll et al. [5] demonstrated that the generation of citrulline through glutamine represented only $13 \%$ of the intestinal metabolism [5-9]. The characteristic of citrulline is that it is absorbed completely without any hepatic consumption [10], suggesting that it could be a better supplementation choice $[6,10]$.

Rougé and des Robert [9] administrated oral citrulline in a dose of $0.18 \mathrm{~g} / \mathrm{kg} /$ day and observed that neither urinary arginine, plasmatic urea nor excretion of nitrates were altered, suggesting that this supplementation was associated with an increased nitrogen balance, and an anabolic effect. In this study plasmatic citrulline increased 5 times (490\%) with oral supplementation while less than $1 \%$ of the citrulline was excreted in the urine, implying that the oral route has good absorption [11, 12].

A comparison of arginine and citrulline supplementation in patients with diastolic HF patients and pulmonary hypertension from our clinic showed that after 8 weeks of treatment systolic pulmonary pressure diminished, and right ventricular ejection fraction (RVEF) rose. Better results were obtained with citrulline [13].

Rector et al. [14] demonstrated that patients with $\mathrm{HF}$ and left ventricular ejection fraction (LVEF) of $18 \pm 5 \%$ who received oral L-arginine (5.6-12.6 g/day) for 6 weeks achieved a significant improvement in blood flow in one extremity and lower production of endothelin-1. Likewise, when Böger and Bode-Böger [15] administered $8 \mathrm{~g}$ /day of L-arginine for 4 weeks to patients with $\mathrm{HF}$ and $\mathrm{LVEF}$ of $19 \pm 9 \%$, they found an $8.8 \%$ improvement in vasodilatation in the radial artery.
The objective of the present study is to evaluate the effect of L-citrulline supplementation on functional class, ejection fraction and peripheral blood flow in patients with systolic HF. This will complement our previous work on the effect of NO precursors on diastolic HF and pulmonary hypertension.

\section{Hypothesis}

In systolic HF patients oral L-citrulline supplementation will improve endothelial and ventricular function.

\section{Methods}

\section{Study population}

From February to July of 2011 outpatients receiving treatment in the Heart Failure Clinic at the Instituto Nacional de Ciencias Médicas y Nutrición "Salvador Zubirán" were recruited. Subjects included men and non-pregnant women more than 18 years of age with a confirmed diagnosis of systolic HF in NYHA functional classes I and II. Systolic HF was defined as signs and symptoms of HF and LVEF of $\leq 35 \%$ measured by echocardiogram and/or radioisotopic ventriculography. Patients were excluded if they had myocardial ischemia susceptible to revascularization, recent myocardial infarction, unstable angina or a myocardial revascularization procedure, including percutaneous transluminal coronary angioplasty and aorto-coronary by-pass grafting, a cerebrovascular event within the last 3 months, dysfunctional prosthetic valve, obstructive or nonobstructive hypertrophic cardiomyopathy, uncorrected congenital heart disease, active myocarditis, a history of resuscitation after sudden death, or severe arrhythmias.

All patients received standard HF therapy (diuretics, ACE inhibitors, angiotensin II antagonists, aldosterone receptor blockers, digitalis and beta-adrenoreceptor blockers), according their maximum doses tolerated.

After baseline measurements, patients were randomly assigned to one of the following groups: 1) control group, without supplementation and optimal pharmacological treatment $(\mathrm{n}=15)$ or 2$)$ experimental group $(n=20)$ who received $3 \mathrm{~g} /$ day of L-citrulline malate powder in two doses of $1.5 \mathrm{~g}$ in addition to conventional therapy considering the dose suggested in the literature [9, 11-13]. Investigators and study personnel with any participation on the study were blinded to the characteristics, evaluation of patient and treatment group allocation. Supplements were provided as weighed daily 
amounts to be taken as a drink mixed with juice or water throughout the day, during the 4-month treatment period. Compliance was evaluated using the supplement consumption records kept daily by patients, and by collecting the empty containers.

The study was approved by the Institutional Ethics Committee of biomedical Research in $\mathrm{Hu}-$ mans of the INCMNSZ, and all patients signed informed consent forms.

\section{Radio-isotopic ventriculography technique}

Left and right ventricular functions at rest and in exercise were evaluated by radio-isotopic ventriculography, which is one of the simplest techniques to provide an initial functional evaluation among HF patients [16-18].

The procedure was performed at rest in the supine position using in vivo red blood cell labeling with technetium-99m by standard methods [19].

The patients were required to fast for $4 \mathrm{~h}$ prior to the study and refrain from caffeine for $24 \mathrm{~h}$. They were injected with $40 \mathrm{mg}$ of stannous pyrophosphate in $1.5 \mathrm{~mL}$ saline. Ten minutes later, the patients were positioned on the bed of the camera with the detector in the right anterior oblique (RAO) position. A rapid bolus of $20 \mathrm{mCi}$ technetium-99m pertechnetate was given intravenously, together with the start of a list mode acquisition. Following the first pass study and after time for equilibration in the blood volume, a standard gated cardiac blood pool study was acquired in the left anterior oblique (LAO) and left lateral projections. The $\mathrm{R}$ to $\mathrm{R}$ interval was divided into 16 frames, not greater than $0.04 \mathrm{~s}$ in length. Multigated acquisition was recorded for $900 \mathrm{~s}$ in a $64 \times 64$ matrix. Quantization was performed on the LAO view. Processing and measurements were made according to the American Society of Nuclear Cardiology Society guidelines.

Also, all participants were studied with a multistage exercise treadmill test according to the Bruce modified protocol. Systolic and diastolic blood pressures were recorded by cuff when the subject was standing immediately before testing and during the last minute of the last exercise stage. Subjects exercised until reaching and age-specific target heart rate or the development of symptoms necessitating termination of the test.

\section{Photoplethysmography}

A baseline digital photoplethysmographic wave was recorded for $30 \mathrm{~s}$. The forearm was then compressed with a sphygmomanometer cuff for $5 \mathrm{~min}$ using a pressure of $30 \mathrm{~mm} \mathrm{Hg}$ above the systolic arterial pressure recorded (ischemic phase). The compression was then released and the digital photoplethysmographic wave was recorded for $120 \mathrm{~s}$. The wave was analyzed at $30 \mathrm{~s}$ intervals for comparison with the baseline values. The most representative waves were selected from the recording of each interval, and the maximum amplitude time (MAT) and total time (TT) were measured in order to calculate the MAT/TT index. A MAT/TT index of less than 30 was considered normal, as proposed in other studies [20,21].

All evaluations were performed at the beginning and 4 months after initiating supplementation by a cardiologist who was blinded to the patient's study group.

\section{Statistical analysis}

Continuous variables were expressed as mean \pm \pm standard deviation (SD), and categorical variables as absolute and relative frequency. For comparisons between the 2 groups at baseline $\chi^{2}$ or Fisher's exact test for categorical and the Mann-Whitney U test for continuous variables were used. To compare the changes from baseline to 4 months between the groups, percentage of change between the initial and final measurements was calculated and Mann-Whitney U test was used. A $p$ value of $<0.05$ was considered statistically significant. All analyses were performed using a commercially available package (SPSS for Windows, version 17.0 SPSS Inc.).

\section{Results}

Three of the 38 patients recruited were eliminated, one from the citrulline group because of gastrointestinal intolerance, and two from the control group because they left the study. Thus, 20 patients constituted the citrulline group and 15 the control group.

Table 1 shows the baseline clinical characteristics of both groups. The main co-morbidities were hypertension, diabetes mellitus, and ischemic heart disease. The hemodynamic baseline characteristics appear in Table 2.

The comparisons between the results obtained after the intervention in each group are presented in Table 3. In the group receiving L-citrulline increases in the LVEF at rest of $20.3 \%$ and $12.7 \%$ with effort, as well as the RVEF at rest of $15.10 \%$ and $14.88 \%$ with effort are evident. These phenomena were not observed in the control group, in which LVEF decreased $6.47 \%$ at rest and $12.6 \%$ with effort, and RVEF decreased $10.7 \%$.

An improvement in functional class occurred in $35 \%$ of the patients in the experimental group $(\mathrm{p}=0.016)$. Patients reported less dyspnea, while 
Table 1. Clinical baseline characteristics of study groups.

\begin{tabular}{lccc}
\hline Variable & L-citrulline group (n=20) & Control group (n= 15) & $\mathbf{P}$ \\
\hline Age (years) & $68.2 \pm 9.3$ & $65.8 \pm 9.5$ & 0.36 \\
Gender (women/men) & $4 / 16$ & $7 / 8$ & 0.16 \\
New York Heart Association I & $10(50 \%)$ & $11(73.3 \%)$ & $4(26.7 \%)$ \\
New York Heart Association II & $10(50 \%)$ & & 0.42 \\
Co-morbidities: & & $13(86.7 \%)$ & 0.25 \\
Systemic hypertension & $14(70 \%)$ & $10(66.7 \%)$ & 0.85 \\
Ischemic heart disease & $17(85 \%)$ & $8(53.3 \%)$ & 0.03 \\
Diabetes mellitus & $10(50 \%)$ & $1(6.7 \%)$ & 1.00 \\
Dyslipidemia & $11(55 \%)$ & $3(20 \%)$ & 1.00 \\
Atrial fibrillation & $4(20 \%)$ & $3(20 \%)$ & \\
Hypothyroidism & $3(15 \%)$ & & 0.41 \\
Treatment: & & $4(26.7 \%)$ & 0.63 \\
Angiotensin converting enzyme inhibitor & $8(40 \%)$ & $7(46.7 \%)$ & 0.37 \\
Angiotensin receptor antagonist & $11(55 \%)$ & $11(73.3 \%)$ & 0.69 \\
Beta-blocker & $18(90 \%)$ & $10(66.7 \%)$ & 0.31 \\
Aspirin & $12(60 \%)$ & $7(46.7 \%)$ & 0.49 \\
Loop diuretic & $6(30 \%)$ & $5(33.3 \%)$ & 0.18 \\
Statins & $9(45 \%)$ & $8(53.3 \%)$ & 1.00 \\
Digitalis & $15(75 \%)$ & $2(13.3 \%)$ & 0.54 \\
Amiodarone & $2(10 \%)$ & $6(40 \%)$ & \\
Nitrates & $6(30 \%)$ & & \\
\hline
\end{tabular}

Table 2. Hemodynamic baseline characteristics of study groups.

\begin{tabular}{lccc}
\hline Variable & L-citrulline group $(\mathbf{n}=\mathbf{2 0})$ & Control group $(\mathbf{n}=\mathbf{1 5})$ & $\mathbf{P}$ \\
\hline Systolic pressure $[\mathrm{mm} \mathrm{Hg}]$ & $113 \pm 17.2$ & $118 \pm 15.8$ & 0.46 \\
Diastolic pressure [mm Hg] & $70.3 \pm 11.5$ & $76.7 \pm 11.0$ & 0.93 \\
Rest left ventricular ejection fraction [\%] & $22.11 \pm 6.7$ & $29.53 \pm 9.2$ & 0.02 \\
Rest right ventricular ejection fraction [\%] & $32.0 \pm 14.4$ & $29.0 \pm 11.2$ & 0.76 \\
Stress left ventricular ejection fraction [\%] & $24.4 \pm 8.6$ & $34.0 \pm 9.1$ & 0.005 \\
Stress right ventricular ejection fraction [\%] & $32.9 \pm 11.7$ & $27.4 \pm 13.9$ & 0.17 \\
Maximum amplitude time/total time index [\%] & $28.2 \pm 9.1$ & $29.07 \pm 7.11$ & 0.43 \\
\hline
\end{tabular}

Table 3. Comparison of changes in blood pressure, cardiac and endothelial function between study groups.

\begin{tabular}{lccc}
\hline Percentage of change & L-citrulline group & Control group & P (\% change) \\
\hline Systolic blood pressure [\%] & $-2.02 \pm 9.36$ & $7.08 \pm 17.08$ & 0.15 \\
Diastolic blood pressure [\%] & $-3.22 \pm 14.01$ & $-1.42 \pm 14.12$ & 0.99 \\
Rest left ventricular ejection fraction [\%] & $+20.30 \pm 40.32$ & $-6.47 \pm 18.08$ & 0.03 \\
Rest right ventricular ejection fraction [\%] & $+15.10 \pm 41.80$ & $-10.72 \pm 42.2$ & 0.09 \\
Stress left ventricular ejection fraction [\%] & $+12.71 \pm 32.6$ & $-12.6 \pm 19.8$ & 0.04 \\
Stress right ventricular ejection fraction [\%] & $+14.88 \pm 33.7$ & $1.80 \pm 45.62$ & 0.22 \\
Maximum amplitude time/total time index [\%] & $-23.13 \pm 21.07$ & $15.99 \pm 25.4$ & $<0.001$ \\
\hline
\end{tabular}


in the control group there was no change, except for 2 patients who went from NYHA II to III, without statistical significance.

The MAT/TT index decreased $23.3 \%$ in experimental group compared to the control group.

\section{Discussion}

$\mathrm{L}$-arginine is a precursor of citrulline that at concentrations of $0.5-1 \mathrm{mmol} / \mathrm{L}$ acts as a scavenger of reactive oxygen species and reduces lipid peroxidation and $\mathrm{O}_{2}$ release from endothelial cells [22]. At high concentrations it regulates the binding of macromolecules to red blood cells, decreasing blood viscosity [23].

Several NO-independent actions of L-arginine have been described: regulation of $\mathrm{pH}$ (intracellular and intravascular); contribution to the depolarization of endothelial cell membranes; antioxidant properties; decrease of blood viscosity; precursor for the synthesis of proteins, urea, polyamines and other amino acids; stimulation of the release of hormones such as insulin, glucagon, prolactin and growth hormone; stimulation of fibrinolysis; decreased formation of thromboxane B2; inhibition of the platelet-fibrin complex; enhanced plasmin generation and enhanced fibrin degradation $[24,25]$. It is an ACE inhibitor that contributes to the regulation of blood pressure [26].

The search for new alternatives to improve clinical condition in patients with $\mathrm{HF}$ and optimize pharmacological treatment opens lines of investigation that attempt to delay ventricular deterioration over time. In this study we found significant changes in functional class and LVEF after citrulline supplementation compared to the control group.

A prior study from our clinic [13] also found a MAT/TT index reduction that could be interpreted as improved distribution of peripheral blood flow. This could be attributed to a constant supply of the NO precursor, which probably maintained vasodilatation independent of endothelial synthesis, reducing friction stress. Since we observed changes in both pre-ischemic and post-ischemic periods during the test, it could indicate improved vascular reactivity, as Rector et al. [14] and Böger and Bode-Böger [15] postulated when they measured blood flow in extremities, and reduced resistance affecting left ventricular ejection effort.

Our findings let us suppose there is better peripheral tissue perfusion, particularly of muscle mass, resulting in a better functional class. While this study cannot determine the exact mechanism of citrulline, it certainly demonstrates a positive effect on endothelial function. This peripheral vasodilatation probably reduces the difficulty to ventricular ejection allowing them to be more efficient.

Perivascular delivery of L-arginine reduced vascular remodeling and neointimal thickening after balloon dilatation of rabbit coronary artery [27]. In addition, intravenous infusion of L-arginine enhanced cardiac performance in patients with severe congestive $\mathrm{HF}$, mainly by affecting systemic vascular resistance [28] increasing maximum vasodilatation during reactive hyperemia and reducing peripheral resistance in patients with $\operatorname{HF}[29,30]$. Although there were differences between the groups on some hemodynamic baseline characteristics, the improvement on patients' percentage of change after citrulline supplementation in contrast with those on control group, especially in stress LVEF in a short period, could be explained with an improvement in stress RVEF, increasing with this the left ventricle filling volume, but with worse peripheral vasodilatation (increase in MAT/TT index and systolic blood pressure) and hence decreasing the ejection fraction on stress.

Supplementation based on citrulline seems to be the optimal choice, since as Van de Poll et al. [5] and Rougé et al. [9] described; the lack of hepatic metabolism does not limit the dose and could represent an advantage. Citrulline can be an important adjuvant in the treatment of patients with compensated systolic HF. Future investigations are required to increase follow-up and the number of subjects to evaluate the long term effects.

\section{Limitations of the study}

This study is limited both non-placebo-controlled open label design, and by the small sample size

\section{Conclusions}

The supplementation of citrulline during 4 months in patients with systolic HF improved functional class, LVEF at rest and effort, and vasodilatation independent of endothelial function. So citrulline can be an important co-adjuvant in the treatment of patients with systolic HF.

Conflict of interest: none declared

\section{References}

1. Dickstein K. ESC guidelines for the diagnosis and treatment of acute and chronic heart failure 2008. Eur Heart J, 2008; 29: 2388-2442. 
2. Méndez A. Fisiopatología de la insuficiencia cardiaca. Arch Cardiol Mex, 2006; 76 (S2): 182-187.

3. Libby P, Bonow RO, Mann DL, Zipes DP eds. Braunwald's heart disease: A textbook of cardiovascular medicine. $8^{\text {th }}$ Ed. Elsevier, USA 2007: 234-255.

4. Duarte J, Espinosa R. Óxido nítrico: metabolismo e implicaciones clínicas. Med Int Mex, 2008; 24: 397-406.

5. Van de Poll M, Siroen M, van Leeuwen PA et al. Interorgan amino acid exchange in humans: consequences for arginine and citrulline metabolism. Am J Clin Nutr, 2007; 85: 167-172.

6. Brosnan M, Brosnan T. Renal arginine metabolism. J Nutr, 2004; 134: 2791S-2795S.

7. Romero M, Platt D, Caldwell R. Therapeutic use of citrulline in cardiovascular disease. Cardiovasc Drug Rev, 2006; 24: 275-290.

8. Flam B, Eichler D. Endothelial nitric oxide production is tightly coupled to the citrulline-NO cycle. Nitric Oxide, 2007; 17: 115-121.

9. Rougé C, Des Robert C. Manipulation of citrulline availability in humans. Am J Physiol Gastrointest Liver Physiol, 2007; 293: G1061-G1067.

10. Moinard C, Cynober L. Citrulline: a new player in the control of nitrogen homeostasis. J Nutr, 2007; 137: 1621S-1625S.

11. Figueroa A, Trivino J. Oral L-citrulline supplementation attenuates blood pressure response to cold pressor test in young men. Am J Hypertens, 2010; 23: 12-16.

12. Heffernan K, Fahs C, Ranadive S. L-arginine as a nutritional prophylaxis against vascular endothelial dysfunction with aging. J Cardiovasc Pharmacol Theraphy, 2010; 15: 17-23.

13. Orea-Tejeda A, Orozco-Gutiérrez JJ, Castillo-Martínez L et al. The effect of L-arginine and citrulline on endothelial function in patients in heart failure with preserved ejection fraction. Cardiol J, 2010; 17: 464-470.

14. Rector TS, Bank AJ, Mullen KA et al. Randomized, double-blind, placebo-controlled study of supplemental oral L-arginine in patients with heart failure. Circulation, 1996; 93: 2135-3141.

15. Böger RH, Bode-Böger SM. The clinical pharmacology of L-arginine. Annu Rev Pharmacol Toxicol, 2001; 41: 79-99.

16. Corbett JR, Akinboboye OO, Bacharach SL et al. Equilibrium radionuclide angiocardiography. J Nucl Card, 2006; 13: e56-e79.

17. Schulman DS. Assessment of the right ventricle with radionuclide techniques. J Nucl Cardiol, 1996; 3: 253-264.

18. Zaret BL, Wackers FJ. Nuclear cardiology. Part 2. N Engl J Med, 1993; 329: 855-863.
19. Links JM, Becker LC, Shindledecker JG et al. Measurement of absolute left ventricular volume from gated pool studies. Circulation, 1982; 65: 82-91.

20. Kuvin J, Patel A, Sliney K et al. Assessment of peripheral vascular endothelial function with finger arterial pulse wave amplitude. Am Heart J, 2003; 146: 168-174.

21. Aldama A, Alvarez H, Rodríguez A, Reyes B. Evaluación cualitativa de la morfología de la seńal fotopletismográfica en el diagnóstico de la insuficiencia arterial. Rev Cubana Invest Biomed, 2008; 27 [online].

22. Wascher TC, Posch K, Wallner S, Hermetter A, Kostner GM, Graier WF. Vascular effects of 1-arginine: anything beyond a substrate for the NO-synthase? Biochem Biophys Res Commun, 1997; 234: 35-38.

23. Walter R, Mark M, Reinhart WH. Pharmacological concentrations of arginine influence human whole blood viscosity independent of nitric oxide synthase activity in vitro. Biochem Biophys Res Commun, 2000; 269: 687-691.

24. Tousoulis D, Antoniades Ch, Tentolouris C, Goumas G, Stefanadis Ch, Toutouzas P. L-arginine in cardiovascular disease: dream or reality? Vasc Med, 2002; 7: 203-211.

25. Maxwell AJ, Cooke JP. Cardiovascular effects of L-arginine. Curr Opin Nephrol Hypertens, 1998; 7: 63-70.

26. Higashi $\mathrm{Y}$, Ashima $\mathrm{T}$, Ono $\mathrm{N}$ et al. Intravenous administration of L-arginine inhibits angiotensin-converting enzyme in humans. J Clin Endocrinol Metab, 1995; 80: 2198-202.

27. Bosmans JM, Vrints CJ, Kockx MM, Bult H, Cromheeke KM, Herman AG. Continuous perivascular L-arginine delivery increases total vessel area and reduces neointimal thickening after experimental balloon dilatation. Arterioscler Thromb Vasc Biol, 1999; 19: 767-776.

28. Bocchi EA, Vilella de Moraes AV, Esteves-Filho A et al. L-arginine reduces heart rate and improves hemodynamics in severe congestive heart failure. Clin Cardiol, 2000; 23: 205-210 .

29. Hirooka $\mathrm{Y}$, Imaizumi $\mathrm{T}$, Tagawa $\mathrm{T}$ et al. Effects of $\mathrm{L}$-arginine on impaired acetylcholine-induced and ischemic vasodilation of the forearm in patients with heart failure. Circulation, 1994; 90: 658-668 .

30. Kanaya $\mathrm{Y}$, Nakamura M, Kobayashi $\mathrm{N}$ et al. Effects of L-arginine on lower limb vasodilator reverse and exercise capacity in patients with chronic heart failure. Heart, 1999; 81: 512-517. 